Bull. Austral. Math. Soc.

VOL. 65 (2002) [35-44]

\title{
SEMI-ORTHOGONAL FRAME WAVELETS AND FRAME MULTI-RESOLUTION ANALYSES
}

\author{
Hong Oh Kim, Rae Young Kim and Jae Kun Lim
}

\begin{abstract}
We first characterise semi-orthogonal frame wavelets by generalising the characterisation of orthonormal wavelets. We then characterise those semi-orthogonal frame wavelets that are associated with frame multi-resolution analyses. This is a generalisation of a result of Wang and another result of Papadakis. Finally, we illustrate our results by an example.
\end{abstract}

\section{INTRODUCTION}

It is well-known that most wavelets are associated with multi-resolution analyses, whereas there exist some 'pathological' wavelets that are not associated with any multiresolution analyses. We are going to be more clear about what we mean. Let $\psi \in L^{2}(\mathbb{R})$ be an orthonormal wavelet if it generates a wavelet orthonormal basis, that is, $\left\{\psi_{j k}:=\right.$ $\left.D^{j} T_{k} \psi: j, k \in \mathbb{Z}\right\}$ is an orthonormal basis of $L^{2}(\mathbb{R})$, where $D: L^{2}(\mathbb{R}) \rightarrow L^{2}(\mathbb{R})$ is the unitary dilation operator defined by $D f(x):=2^{1 / 2} f(2 x)$, and $T_{t}$ is the translation operator defined by $T_{t} f(x):=f(x-t)$ for $t \in \mathbb{R}$. The following useful commutation relation holds:

$$
D^{n} T_{t}=T_{2^{-n_{t}}} D^{n}, \text { or } \quad T_{t} D^{n}=D^{n} T_{2^{n} t}
$$

We recall the characterisation of orthonormal wavelets in $[5,6,7,14]$ :

THEOREM 1. $\psi \in L^{2}(\mathbb{R})$ is an orthonormal wavelet if and only if

(a) $\|\psi\|_{L^{2}(\mathbb{R})}=1$;

(b) $\sum_{j \in \mathbf{Z}}\left|\widehat{\psi}\left(2^{j} x\right)\right|^{2}=1$ for almost everywhere $x \in \mathbb{R}$;

(c) $\sum_{j=0}^{\infty} \widehat{\psi}\left(2^{j} x\right) \overline{\widehat{\psi}}\left(2^{j}(x+2 m \pi)\right)=0$ for almost everywhere $x \in \mathbb{R}, m \in 2 \mathbb{Z}+1$.

We use the following form of the Fourier transform: For $f \in L^{1}(\mathbb{R}) \cap L^{2}(\mathbb{R})$ define $\hat{f}(x):=\int_{\mathbb{R}} f(t) e^{-i x t} d t$ and extend the Fourier transform $\wedge$ to be $\sqrt{2 \pi}$ times a unitary operator from $L^{2}(\mathbb{R})$ onto $L^{2}(\mathbb{R})$. The most efficient way to construct an orthonormal wavelet is to construct it from an orthonormal multi-resolution analysis ([7]).

Received 4th April, 2001

Copyright Clearance Centre, Inc. Serial-fee code: 0004-9727/02 \$A2.00+0.00. 
Definition 2: A family $\left\{V_{j}\right\}_{j \in Z}$ of closed subspaces of $L^{2}(\mathbb{R})$ is said to be a multiresolution analysis if

(i) $V_{j} \subset V_{j+1}$ for each $j \in \mathbb{Z}$;

(ii) $D\left(V_{j}\right)=V_{j+1}$ and $T_{1}\left(V_{0}\right)=V_{0}$;

(iii) $\overline{\bigcup_{j \in \mathbf{Z}} V_{j}}=L^{2}(\mathbb{R})$ and $\bigcap_{j \in \mathbf{Z}} V_{j}=\{0\}$;

(iv) There exists $\varphi \in V_{0}$ such that $\left\{T_{k} \varphi: k \in \mathbb{Z}\right\}$ is an orthonormal basis for $V_{0}$.

It is well-known that given a multi-resolution analysis there exists $\psi \in V_{1} \ominus V_{0}$ such that $\left\{\psi_{j k}: j, k \in \mathbb{Z}\right\}$ is an orthonormal basis for $L^{2}(\mathbb{R})([7])$. On the other hand, suppose that an orthonormal wavelet $\psi$ is given. Let $V_{j}:=\overline{\operatorname{span}}\left\{\psi_{l k}: k \in \mathbb{Z}, l<j\right\}$ for $j \in \mathbb{Z}$. Then it is easy to see that if there exists $\varphi \in V_{0}$, called the scaling function, such that $\left\{T_{k} \varphi: k \in \mathbb{Z}\right\}$ is an orthonormal basis for $V_{0}$, then $\left\{V_{j}\right\}_{j \in \mathbf{Z}}$ is a multi-resolution analysis. In this case we say that $\psi$ is associated with a multi-resolution analysis. It is established that most 'nice' wavelets are associated with multi-resolution analyses [7, Chapter 7]. For example, any compactly supported orthonormal wavelet is associated with a multiresolution analysis ([7, Corollary 3.15, Chapter 7]). On the other hand, there are some 'pathological' orthonormal wavelets that are not associated with multi-resolution analyses ([14, p. 77], [6]). Hernández and Weiss along with Wang ([7, 14]) characterised those orthonormal wavelets that are associated with multi-resolution analyses. Let $\mathbb{T}$ denote the circle group which can be identified with $[-\pi, \pi)$.

THEOREM 3. An orthonormal wavelet $\psi$ is associated with a multi-resolution analysis if and only if $\sum_{j=1}^{\infty} \sum_{k \in \mathbb{Z}}\left|\widehat{\psi}\left(2^{j}(x+2 k \pi)\right)\right|^{2}=1$ for almost every $x \in \mathbb{T}$.

A sequence $\left\{f_{i}: i \in I\right\}$ of elements of a Hilbert space $\mathcal{H}$ is said to be a frame for $\mathcal{H}$ if there exist positive constants $A$ and $B$ such that for each $f \in \mathcal{H} A \leqslant \sum_{i}\left|\left\langle f, f_{i}\right\rangle\right|^{2} \leqslant B$. If $\left\{f_{i}: i \in I\right\}$ is a frame for $\mathcal{H}$, then there exists another frame $\left\{\tilde{f}_{i}: i \in I\right\}$ for $\mathcal{H}$, called the dual frame, such that for any $f \in \mathcal{H} f=\sum_{i}\left\langle f, \tilde{f}_{i}\right\rangle f_{i}$. Hence we can expand any vector by a frame. Moreover, unlike orthonormal basis, a frame can be redundant. In some situations this redundancy is positively sought after. See [7] for more details on frames. Papadakis ([13]) proved the following.

THEOREM 4. Any orthonormal wavelet $\psi$ is associated with a generalised multiresolution analysis in the sense that there exists a countable (finite or countably infinite) subset $\Phi$ of $V_{0}$ such that $\left\{T_{k} \varphi: k \in \mathbb{Z}, \varphi \in \Phi\right\}$ is a frame for $V_{0}$.

In this paper we generalise Theorem 1, Theorem 3 and Theorem 4 to semi-orthogonal wavelet frames and frame multi-resolution analyses (see Theorems 7 and 11). First, let us introduce some definitions in order to clarify what we are going to show. $\psi \in L^{2}(\mathbb{R})$ is said to be a frame wavelet if it generates a wavelet frame for $L^{2}(\mathbb{R})$, that is, $\left\{\psi_{j k}: j, k \in \mathbb{Z}\right\}$ is a frame for $L^{2}(\mathbb{R})$. It is said to be a semi-orthogonal frame wavelet if the wavelet frame 
it generates is semi-orthogonal in the sense that $\left\langle\psi_{j k}, \psi_{l m}\right\rangle=0$ if $j \neq l .\left\{V_{j}\right\}_{j \in \mathbf{Z}}$ is said to be a frame multi-resolution analysis if Condition (iv) in Definition 2 is replaced by the following.

(iv)' There exists $\varphi \in V_{0}$ such that $\left\{T_{k} \varphi: k \in \mathbb{Z}\right\}$ is a frame for $V_{0}$.

It is said to be a finite frame multi-resolution analysis if Condition (iv) in Definition 2 is replaced by the following.

(iv)" There exists a finite subset $\Phi \subset V_{0}$ such that $\left\{T_{k} \varphi: k \in \mathbb{Z}, \varphi \in \Phi\right\}$ is a frame for $V_{0}$.

If $\Phi$ is countably infinite we say that $\left\{V_{j}\right\}_{j \in \mathbb{Z}}$ is an infinite frame multi-resolution analysis. Frame multi-resolution analyses were introduced in [1] with an intention to apply the theory to analyse narrow band signals. The fundamental existence problem concerning frame multi-resolution analyses was solved independently in [2] and [10], and some extension of the theory can be found in [11].

In Section 2 we generalise Theorem 1 in the sense that we find equivalent conditions for $\psi$ to be a semi-orthogonal frame wavelet. Then a generalisation of both Theorem 3 and Theorem 4 is presented in Section 3. The idea is to apply shift-invariant space theory $([3,4,8])$ to the problem of association of a wavelet with a multi-resolution analysis. Our solution to the problem of the association of a Riesz wavelet, that is, $\left\{\psi_{j k}: j, k \in \mathbb{Z}\right\}$ is a Riesz basis of $L^{2}(\mathbb{R})$, with a multi-resolution analysis is reported in [12]. Finally, we illustrate our results by an example.

\section{Semi-orthogonal frame WAVELETS}

We first characterise semi-orthogonal frame wavelets as a generalisation of the characterisation of orthonormal wavelets by Gripenberg ([5]), Ha, Kang, Lee and Seo ([6]), and Hernández and Weiss, and also Wang $([\mathbf{7}, \mathbf{1 4}])$. The following two propositions are well known. See [7, Theorem 1.6, Chapter 7] and [9, Theorem A3], respectively.

Proposition 5. Let $\psi \in L^{2}(\mathbb{R})$. Then $\left\{\psi_{j, k}: j, k \in \mathbb{Z}\right\}$ is a tight frame with frame bound 1 for $L^{2}(\mathbb{R})$, that is,

$$
\sum_{j, k}\left|\left\langle f, \psi_{j, k}\right\rangle\right|^{2}=\|f\|^{2}, \text { for all } f \in L^{2}(\mathbb{R}),
$$

if and only if $\psi$ satisfies (b) and (c) of Theorem 1.

PRoposition 6. Let $\psi \in L^{2}(\mathbb{R})$ and let $W_{0}=\overline{\operatorname{span}}\left\{\psi_{0, k}: k \in \mathbb{Z}\right\}$. Then $\left\{\psi_{0, k}: k \in \mathbb{Z}\right\}$ is a frame for $W_{0}$ if and only if there exist positive constants $A, B$ such that

$$
A \leqslant\left\|\hat{\psi}_{\| x}\right\|_{\ell^{2}(\mathcal{Z})}^{2} \leqslant B \text { for almost every } x \in \mathbb{T} \backslash N,
$$

where $\widehat{\psi}_{\| x}:=(\widehat{\psi}(x-2 \pi k))_{k \in \mathbb{Z}}$ and $N:=\left\{x \in \mathbb{T}: \widehat{\psi}_{\| x}=0\right\}$. In this case, $A$ and $B$ are frame bounds for $\left\{\psi_{0, k}: k \in \mathbb{Z}\right\}$. 
Now, we state and prove our characterisation of semi-orthogonal frame wavelets.

THEOREM 7. Let $\psi \in L^{2}(\mathbb{R})$ and define $\psi^{*}$ by

$$
\widehat{\psi^{*}}(x):= \begin{cases}\frac{\widehat{\psi}(x)}{\left\|\widehat{\psi}_{\| x}\right\|_{\ell^{2}(\mathbb{Z})}}, & \text { if } \widehat{\psi}_{\| x} \neq 0 \\ 0, & \text { otherwise }\end{cases}
$$

Then the following statements are equivalent:

(a) $\left\{\psi_{j, k}: j, k \in \mathbb{Z}\right\}$ is a semi-orthogonal frame wavelet with frame bounds $A$ and $B$;

(b) There exist positive constants A,B such that $\psi$ satisfies (3) and

$$
\begin{gathered}
\sum_{j \in Z}\left|\widehat{\psi^{*}}\left(2^{j} x\right)\right|^{2}=1, \text { for almost every } x \in \mathbb{R} \\
\sum_{j \geqslant 0} \widehat{\psi^{*}}\left(2^{j} x\right) \widehat{\widehat{\psi^{*}}}\left(2^{j}(x+2 p \pi)\right)=0, \text { for almost every } x \in \mathbb{R}, p \in 2 \mathbb{Z}+1 ;
\end{gathered}
$$

(c) There exist positive constants $A, B$ such that $\psi$ satisfies (3), (5) and

$$
\begin{gathered}
\sum_{k \in \mathbb{Z}} \widehat{\psi}(x+2 k \pi) \overline{\widehat{\psi}}\left(2^{j}(x+2 k \pi)\right)=0, \text { almost every } x \in \mathbb{R}, j \geqslant 1, \\
A \leqslant \sum_{j \in \mathbb{Z}}\left|\widehat{\psi}\left(2^{j} x\right)\right|^{2} \leqslant B, \text { for almost every } x \in \mathbb{R} .
\end{gathered}
$$

PRoOF: Let $W_{j}=\overline{\operatorname{span}}\left\{\psi_{j, k}: k \in \mathbb{Z}\right\}$ and $W_{j}^{*}=\overline{\operatorname{span}}\left\{\psi_{j, k}^{*}: k \in \mathbb{Z}\right\}$. Note that $W_{j}=W_{j}^{*}$.

(a) $\Rightarrow(\mathrm{b})$ : Suppose that $\psi$ is a semi-orthogonal frame wavelet with frame bounds $A$ and $B$, that is,

$$
A\|f\|^{2} \leqslant \sum_{j, k \in \mathbb{Z}}\left|\left\langle f, \psi_{j, k}\right\rangle\right|^{2} \leqslant B\|f\|^{2}, f \in L^{2}(\mathbb{R}) .
$$

Take $f \in W_{0}$. Since $W_{j} \perp W_{j^{\prime}}$ for $j \neq j^{\prime}$ by the semi-orthogonality, we have

$$
A\|f\|^{2} \leqslant \sum_{k \in \mathbf{Z}}\left|\left\langle f, \psi_{0, k}\right\rangle\right|^{2} \leqslant B\|f\|^{2},
$$

which is equivalent to (3) by Proposition 6.

Since $W_{j}^{*}$ 's are orthogonal to each other and

$$
\sum_{k \in \mathbb{Z}}\left|\widehat{\psi^{*}}(x-2 \pi k)\right|^{2}=1, \text { for almost everywhere } x \in \mathbb{T} \backslash N
$$


$\left\{\psi_{j, k}^{*}\right\}_{j, k \in \mathbb{Z}}$ is a tight frame with frame bound 1 for $L^{2}(\mathbb{R})$. Hence (4) and (5) are satisfied by Proposition 5 .

(b) $\Rightarrow$ (c): From (3), we have

$$
\frac{1}{B}|\widehat{\psi}(x)|^{2} \leqslant\left|\widehat{\psi^{*}}(x)\right|^{2} \leqslant \frac{1}{A}|\widehat{\psi}(x)|^{2}, \text { for almost every } x \in \mathbb{R} .
$$

Hence, by Condition (4) we have

$$
A \leqslant \sum_{j \in \mathbb{Z}}\left|\widehat{\psi}\left(2^{j} x\right)\right|^{2} \leqslant B, \text { for almost everywhere } x \in \mathbb{R},
$$

which shows (7). From the definition of $\psi^{*}$, we see that $\left\{\psi_{0, k}^{*}: k \in \mathbb{Z}\right\}$ is a tight frame for $W_{0}^{*}$ with frame bound 1 . By Proposition $5,\left\{\psi_{j, k}^{*}: j, k \in \mathbb{Z}\right\}$ is also a tight frame with frame bound 1 for $L^{2}(\mathbb{R})$. Since $\psi^{*}$ is in $W_{0}$, it follows from the tightness of both $\left\{\psi_{0, k}^{*}: k \in \mathbb{Z}\right\}$ and $\left\{\psi_{j, k}^{*}: j, k \in \mathbb{Z}\right\}$ that

$$
\begin{aligned}
\left\|\psi^{*}\right\|_{L^{2}(\mathbb{R})}^{2} & =\sum_{j, k \in \mathbb{Z}}\left|\left\langle\psi^{*}, \psi_{j, k}^{*}\right\rangle\right|^{2} \\
& =\sum_{k \in \mathbb{Z}}\left|\left\langle\psi^{*}, \psi_{0, k}^{*}\right\rangle\right|^{2} .
\end{aligned}
$$

Therefore, $\left\langle\psi^{*}, \psi_{j, k}^{*}\right\rangle=0$ for $j \neq 0$. We argue as in $[7$, Section 3.1] below:

$$
\begin{aligned}
0=\left\langle\psi^{*}, \psi_{j, k}^{*}\right\rangle & =\frac{1}{2 \pi} \int_{\mathbb{R}} \widehat{\psi^{*}}(x) 2^{-j / 2} \overline{\widehat{\psi^{*}}}\left(2^{-j} x\right) e^{i 2^{-j} k x} d x \\
& =\frac{1}{2 \pi} \int_{\mathbb{R}} \widehat{\psi^{*}}\left(2^{j} x\right) 2^{j / 2} \overline{\widehat{\psi^{*}}}(x) e^{i k x} d x .
\end{aligned}
$$

Thus, we have

$$
\begin{aligned}
0 & =\sum_{l \in \mathbb{Z}} \int_{2 l \pi}^{2(l+1) \pi} \widehat{\psi^{*}}\left(2^{j} x\right) \overline{\widehat{\psi^{*}}}(x) e^{i k x} d x \\
& =\int_{0}^{2 \pi}\left\{\sum_{l \in \mathbb{Z}} \overline{\widehat{\psi^{*}}}(x+2 k \pi) \widehat{\psi^{*}}\left(2^{j}(x+2 k \pi)\right\} e^{i k x} d x\right.
\end{aligned}
$$

for all $k \in \mathbb{Z}$ when $j \geqslant 1$. This shows that

$$
\sum_{l \in \mathbf{Z}} \widehat{\psi^{*}}(x+2 k \pi) \widehat{\widehat{\psi^{*}}}\left(2^{j}(x+2 k \pi)\right)=0, \text { for almost every } x \in \mathbb{R}, j \geqslant 1
$$

Therefore, we have

$$
\sum_{k \in \mathbb{Z}} \widehat{\psi}(x+2 k \pi) \overline{\widehat{\psi}}\left(2^{j}(x+2 k \pi)\right)=\left\|\widehat{\psi}_{\| x}\right\|_{\ell^{2}(\mathbb{Z})}\left\|\widehat{\psi}_{\| 2^{j} x}\right\|_{\ell^{2}(\mathbb{Z})} \sum_{k \in \mathbb{Z}} \widehat{\psi^{*}}(x+2 k \pi) \overline{\psi^{*}}\left(2^{j}(x+2 k \pi)\right)=0
$$

Thus, $\psi$ satisfies Condition (6).

(c) $\Rightarrow(\mathrm{a})$ : Condition (3) implies that $\left\{\psi_{j, k}: k \in \mathbb{Z}\right\}$ is a frame for $W_{j}$ by Proposition 
6 , and Condition (6) shows that $W_{0}$ is orthogonal to $W_{j}$ for $j \neq 0$. By means of change of variables, $\left\langle\psi_{j, k}, \psi_{l, m}\right\rangle=\left\langle\psi_{0, k-2^{j-l} m}, \psi_{l-j, 0}\right\rangle$, from which $W_{j} \perp W_{l}$ follows for $j \neq l$. Therefore, $\left\{\psi_{j, k}: j, k \in \mathbb{Z}\right\}$ is a frame of $W:=\overline{\operatorname{span}}\left\{\psi_{j, k}: j, k \in \mathbb{Z}\right\}$. We claim that $W=L^{2}(\mathbb{R})$. It suffices to show that $\left\{\psi_{j, k}^{*}\right\}$ is a frame for $L^{2}(\mathbb{R})$. As in $[7$, Proposition 1.19, Chapter 7],

$$
\begin{aligned}
& \sum_{j, k \in \mathbf{Z}}\left|\left\langle f, \psi_{j, k}^{*}\right\rangle\right|^{2} \\
& =\frac{1}{2 \pi} \int_{-\infty}^{\infty}|\widehat{f}(x)|^{2} \sum_{j \in \mathbf{Z}}\left|\widehat{\psi^{*}}\left(2^{j} x\right)\right|^{2} d x \\
& \quad+\frac{1}{2 \pi} \sum_{n \in \mathbf{Z}} \sum_{p \in 2 \mathbf{Z}+1} \int_{-\infty}^{\infty} \widehat{f}(x) \overline{\widehat{f}}\left(x+2 \pi p 2^{n}\right) \theta_{p}\left(2^{-n} x\right) d x \\
& =\frac{1}{2 \pi} \int_{-\infty}^{\infty}|\widehat{f}(x)|^{2} \sum_{j \in \mathbf{Z}}\left|\widehat{\psi^{*}}\left(2^{j} x\right)\right|^{2} d x
\end{aligned}
$$

since $\theta_{p}(x):=\sum_{l=0}^{\infty} \widehat{\psi^{*}}\left(2^{l} x\right) \overline{\widehat{\psi^{*}}}\left(2^{l}(x+2 p \pi)\right)=0$ by (5). From (7) and (9), we have

$$
A / B \leqslant \sum_{j \in \mathbf{Z}}\left|\widehat{\psi^{*}}\left(2^{j} x\right)\right|^{2} \leqslant B / A
$$

Thus we obtain from (10),

$$
A / B\|f\|^{2} \leqslant \sum_{j, k \in \mathbb{Z}}\left|\left\langle f, \psi_{j, k}^{*}\right\rangle\right|^{2} \leqslant B / A\|f\|^{2} .
$$

That is, $\left\{\psi_{j, k}^{*}\right\}$ is a frame for $L^{2}(\mathbb{R})$ and hence spans $L^{2}(\mathbb{R})$. Therefore, $W=\overline{\cup W_{j}^{*}}=$ $L^{2}(\mathbb{R})$.

\section{Frame multiresolution analyses}

In this section we characterise those semi-orthogonal frame wavelets which are associated with frame multi-resolution analyses. This association problem can best be understood by the theory of shift-invariant spaces. We first introduce briefly those parts of shift-invariant space theory that will be used directly in this paper. The theory has a rich history, and is well-known to approximation theorists. The interested reader may consult $[3,4,8]$ and the references therein. A closed subspace $S$ of $L^{2}(\mathbb{R})$ is said to be shift-invariant if $T_{k} f \in S$ for any $f \in S$ and $k \in \mathbb{Z}$. Let $\Phi \subset L^{2}(\mathbb{R})$. Then $S:=S(\Phi):=\overline{\operatorname{span}}\left\{T_{k} \varphi: \varphi \in \Phi, k \in \mathbb{Z}\right\}$ is clearly shift-invariant. The length of $S$

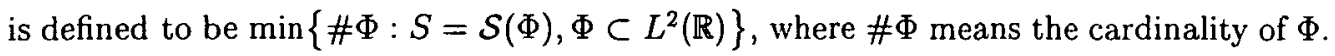
It is established in [3, Section 3] that the length of a shift-invariant subspace of $L^{2}(\mathbb{R})$ is at most countable. For $f \in L^{2}(\mathbb{R})$, let $\widehat{f}_{\| x}:=(\widehat{f}(x-2 \pi k))_{k \in \mathbb{Z}}$, which is in $\ell^{2}(\mathbb{Z})$ for almost every $x \in \mathbb{T}$. For $x \in \mathbb{T}, A \subset L^{2}(\mathbb{R})$ we let $\hat{A}_{\| x}:=\left\{\hat{f}_{\| x}: f \in A\right\}$. 
LEMMA 8. Let $S$ be a shift-invariant subspace of $L^{2}(\mathbb{R})$, and $\lambda$ its length which may be infinite. Then there exists $\Phi \subset L^{2}(\mathbb{R})$, with cardinality $\lambda$, such that $\left\{T_{k} f: k \in\right.$ $\mathbb{Z}, f \in \Phi\}$ is a frame for $S$. Moreover, if $S=\mathcal{S}(\Psi)$ for some $\Psi \subset L^{2}(\mathbb{R})$, then

$$
\begin{aligned}
\lambda & =\operatorname{ess-sup}\left\{\operatorname{dim} \widehat{S}_{\| x}: x \in \mathbb{T}\right\} \\
& =\operatorname{ess-sup}\left\{\operatorname{dim} \overline{\operatorname{span}} \widehat{\Psi}_{\| x x}: x \in \mathbb{T}\right\}
\end{aligned}
$$

ProOF: The first part of the theorem follows from [4, Theorem 3.3] and the remark following it. The equations concerning $\lambda$ follow from [3, Theorem 3.5] and [4, Proposition $1.5]$.

Suppose that $\psi$ generates a semi-orthogonal wavelet frame, that is, $\left\{D^{j} T_{k} \psi: j, k \in\right.$ $\mathbb{R}\}$ is a frame for $L^{2}(\mathbb{R})$ and $\left\langle D^{j} T_{k} \dot{\psi}, D^{l} T_{m} \psi\right\rangle=0$ if $j \neq l$. Let $W_{l}:=\overline{\operatorname{span}}\left\{D^{l} T_{k} \psi: k \in\right.$ $\mathbb{Z}\}$, and $V_{j}:=\bigoplus_{l<j} W_{l}$ for $j, l \in \mathbb{Z}$. Then it is easy to see that $\left\{D^{l} T_{k} \psi: k \in \mathbb{Z}\right\}$ is a frame for $W_{l}$ for each $l \in \mathbb{Z}$, and that $L^{2}(\mathbb{R})=\bigoplus_{l \in \mathbb{Z}} W_{l}$. It is also easy to see that $\psi$ is associated with a frame multi-resolution analysis if and only if there exists $\varphi \in V_{0}$ such that $\left\{T_{k} \varphi: k \in \mathbb{Z}\right\}$ is a frame for $V_{0} ; \psi$ is associated with a finite frame multi-resolution analyses if and only if there exists $\left\{\varphi_{1}, \varphi_{2}, \ldots, \varphi_{n}\right\} \subset V_{0}$ such that $\left\{T_{k} \varphi_{i}: k \in \mathbb{Z}, 1 \leqslant i \leqslant n\right\}$ is a frame for $V_{0} ; \psi$ is associated with an infinite frame multi-resolution analysis if and only if there exists $\left\{\varphi_{i}: i \in \mathbb{N}\right\}$ such that $\left\{T_{k} \varphi_{i}: i \in \mathbb{Z}\right\}$ is a frame for $V_{0}$.

LEMMA $9 . \quad V_{0}$ is shift-invariant.

Proof: First note that $V_{0}^{\perp}=\bigoplus_{l \geqslant 0} W_{l}$. Equation (1) implies that, for each $l \in \mathbb{Z}$, $f \in W_{l}$ if and only if $T_{2^{-l} m} f \in W_{l}$ for each $m \in \mathbb{Z}$, that is, $W_{l}$ is $2^{-l} \mathbb{Z}$-shift-invariant space. In particular, $W_{l}$ is shift-invariant for $l \geqslant 0$. This implies that $V_{0}^{\perp}$ is shift-invariant. Hence so is $V_{0}$ by $[\mathbf{3}$, Corollary 3.4].

Lemma 10. $V_{0}=\mathcal{S}\left(\left\{D^{j} \psi: j<0\right\}\right)$.

Proof: Let $V_{0}^{\prime}:=\mathcal{S}\left(\left\{D^{j} \psi: j<0\right\}\right)$. Note that $V_{0}=\overline{\operatorname{span}}\left\{D^{j} T_{k} \psi: j<0, k \in \mathbb{Z}\right\}$ by the definition of $V_{0}$, and that $V_{0}^{\prime}=\overline{\operatorname{span}}\left\{T_{k} D^{j} \psi: j<0, k \in \mathbb{Z}\right\}=\overline{\operatorname{span}}\left\{D^{j} T_{2_{k}} \psi\right.$ : $j<0, k \in \mathbb{Z}\}$ by the definition of the shift-invariant space and Equation (1). $V_{0}$, however, is shift-invariant by Lemma 9 . Hence

$$
\begin{aligned}
V_{0} & =\overline{\operatorname{span}}\left\{T_{l} D^{j} T_{k} \psi: j<0, k, l \in \mathbb{Z}\right\} \\
& =\overline{\operatorname{span}}\left\{D^{j} T_{2^{j} l+k} \psi: j<0, k, l \in \mathbb{Z}\right\} \\
& =\overline{\operatorname{span}}\left\{D^{j} T_{2^{j} l} \psi: j<0, l \in \mathbb{Z}\right\}=V_{0}^{\prime} .
\end{aligned}
$$

The following theorem gives a generalisation of both [7, Theorem 3.2, Chapter 7] and the main result in [13]. We note that the last part of the following theorem is Theorem 3. 
THEOREM 11. Suppose $\psi$ generates a semi-orthogonal wavelet frame. Let, for $x \in \mathbb{T}$,

$$
D(x):=\operatorname{dim} \overline{\operatorname{span}}\left\{\left(D^{j} \psi\right)_{\| x}^{\wedge}: j<0\right\}
$$

and

$$
\lambda:=\operatorname{ess}-\sup \{D(x): x \in \mathbb{T}\}
$$

which may be infinite. Then $\psi$ is associated with a frame multi-resolution analysis if and only if $\lambda=1$; and it is associated with a finite frame multi-resolution analysis if and only if $\lambda<\infty$. In this case there exists $\left\{\varphi_{1}, \varphi_{2}, \ldots, \varphi_{\lambda}\right\} \subset V_{0}$ such that $\left\{T_{k} \varphi_{i}: k \in \mathbb{Z}\right.$, $1 \leqslant i \leqslant \lambda\}$ is a frame for $V_{0}$. It is associated with an infinite frame multi-resolution analysis if $\lambda=\infty$. Suppose, furthermore, that $\psi$ generates an orthonormal basis. Then

$$
D(x)=\sum_{j=1}^{\infty} \sum_{k \in \mathbf{Z}}\left|\widehat{\psi}\left(2^{j}(x+2 \pi k)\right)\right|^{2}
$$

and it is associated with an orthonormal multi-resolution analysis if and only if $D(x)=1$ for almost everywhere $x \in \mathbb{T}$.

Proof: First note that $\lambda$ is the length of the shift-invariant space $V_{0}$ by Lemma 10 and Lemma 8. Suppose that $\psi$ is associated with a frame multi-resolution analysis. Then there exists $\varphi \in V_{0}$ such that $\left\{T_{k} \varphi: k \in \mathbb{Z}\right\}$ is a frame for $V_{0}$. Hence $V_{0}=\mathcal{S}(\varphi)$. Hence $\lambda=1$ by Lemma 8 . Suppose, on the other hand, that $\lambda=1$. Then there exists $\varphi \in V_{0}$ such that $\left\{T_{k} \varphi: k \in \mathbb{Z}\right\}$ is a frame for $V_{0}$ by Lemma 8 . The statements about finite and infinite frame multi-resolution analyses follow similarly. Now suppose that $\psi$ generates an orthonormal basis. Equation (11) follows from [7, Equation (3.8), Chapter 7]. Suppose that $\psi$ is associated with an orthonormal multi-resolution analysis. Then there exists $\varphi \in V_{0}$ whose translates form an orthonormal basis of $V_{0}$. Hence $V_{0}=\mathcal{S}(\varphi)$. Moreover, $D(x)=\operatorname{dim} \operatorname{span}\left\{\widehat{\varphi}_{\| x}\right\}$ by $\left[4\right.$, Proposition 1.5]. It is well-known that $\left\|\widehat{\varphi}_{\| x}\right\|_{\ell^{2}(\mathbb{Z})}^{2}=2 \pi \neq 0$ for almost every $x \in \mathbb{T}$. Hence $D(x)=1$ for almost every $x \in \mathbb{T}$. Suppose, on the other hand, that $D(x)=1$ for almost every $x \in \mathbb{T}$. Then there exists $\varphi \in V_{0}$ whose translates form an orthonormal basis for $V_{0}$ by [3, Theorem 3.2].

We illustrate our results by considering an example $\psi_{a} \in L^{2}(\mathbb{R})$ defined by $\widehat{\psi_{a}}$ $=\chi_{[-2 a,-a)}+\chi_{[a, 2 a)}$ for $a>0$. That is,

$$
\psi_{a}(x)=(2 / \pi x) \cos (3 a x / 2) \sin (a x / 2)
$$

If $a=\pi, \psi_{\pi}$ is the well-known Shannon wavelet.

For $0<a \leqslant \pi / 2$, we shall show that that $\psi_{a}$ is a semi-orthogonal frame wavelet by checking the conditions in Theorem 7 (b). We see that

$$
\sum_{j \in \mathbf{Z}}\left|\widehat{\psi_{a}}\left(2^{j} x\right)\right|^{2}=\sum_{j \in \mathbf{Z}} \widehat{\psi_{a}}\left(2^{j} x\right)=1, \text { for almost everywhere } x \in \mathbb{R} \text {. }
$$


Since $\widehat{\psi_{a}}(x) \widehat{\widehat{\psi_{a}}}\left(2^{j} x\right)=0$ for $j \geqslant 1$

$$
\sum_{k \in \mathbb{Z}} \widehat{\psi_{a}}(x+2 k \pi) \widehat{\widehat{\psi_{a}}}\left(2^{j}(x+2 k \pi)\right)=0, \text { for almost everywhere } x \in \mathbb{R}, j \geqslant 1
$$

We can check that

$$
\sum_{k \in \mathbf{Z}}\left|\widehat{\psi_{a}}(x+2 k \pi)\right|^{2}=\sum_{k \in \mathbf{Z}} \widehat{\psi_{a}}(x+2 k \pi)=\chi_{\mathbb{T} \backslash N}
$$

where $N=[-\pi,-2 a) \cup[-a, a) \cup[2 a, \pi)$. Finally, we check Condition (5). Let $2^{j} x \in$ $[-2 a,-a) \cup[a, 2 a)$ for $j \geqslant 0$ and let $p \in 2 \mathbb{Z}+1$. If $p \geqslant 1$, then $2^{j} x+2 p 2^{j} \pi \geqslant 2^{j} x+2 \pi \geqslant$ $-2 a+2 \pi \geqslant \pi \geqslant 2 a$. If $p \leqslant-1$, then $2^{j} x+2 p 2^{j} \pi \leqslant 2^{j} x-2 \pi<2 a-2 \pi \leqslant-2 a$. We have

$$
\widehat{\psi_{a}}\left(2^{j} x\right) \widehat{\widehat{\psi_{a}}}\left(2^{j}(x+2 p \pi)\right)=0 \text { for } j \geqslant 0 \text { and } p \in 2 \mathbb{Z}+1
$$

and hence

$$
\sum_{j \geqslant 0} \widehat{\psi_{a}}\left(2^{j} x\right) \widehat{\overline{\psi_{a}}}\left(2^{j}(x+2 p \pi)\right)=0, p \in 2 \mathbb{Z}+1 .
$$

Therefore, we have shown that $\psi_{a}$ is a semi-orthogonal frame wavelet for $0<a \leqslant \pi / 2$ by Theorem 7 . We can also check that $\psi_{a}$ is not a semi-orthogonal frame wavelet if $\pi / 2<a<\pi$ or $a>\pi$ by using Theorem 7 .

Now, we show that $\psi_{a}$ is associated with a frame multi-resolution analyses for $0<$ $a \leqslant \pi / 2$ by applying Theorem 11. If $x \in[-\pi,-a) \cup[a, \pi)$, we see that $\widehat{\psi}\left(2^{j}(x+2 \pi k)\right)=0$ for $k \in \mathbb{Z}$ and $j \geqslant 1$ and so $D(x)=0$. If $x \in[-a, a) \backslash\{0\}$ then $2^{j_{x}} x \in[-2 a,-a) \cup[a, 2 a)$ for some $j_{x} \geqslant 1$ and so $\widehat{\psi}\left(2^{j}(x+2 k \pi)\right)=\delta_{j, j_{x}} \delta_{0, k}$; hence $D(x)=1$. Therefore $\lambda=1$ and so $\psi_{a}$ is associated with a frame multi-resolution analysis by Theorem 11 .

\section{REFERENCES}

[1] J.J. Benedetto and S. Li, 'The theory of multiresolution analysis frames and applications to filter banks', Appl. Comput. Harmon. Anal. 5 (1998), 389-427.

[2] J.J. Benedetto and O.M. Treiber, 'Wavelet frames: multiresolution analysis and extension principle', in Wavelet transforms and time-frequency signal analysis, (L. Debnath, Editor) (Birkhauser, Boston, 2000).

[3] C. de Boor, R. DeVore and A. Ron, 'The structure of finitely generated shift-invariant spaces in $L_{2}\left(\mathbb{R}^{d}\right)^{\prime}, J$. Funct. Anal. 119 (1994), 37-78.

[4] M. Bownik, 'The structure of shift-invariant subspaces of $L^{2}\left(\mathbb{R}^{n}\right)$ ', J. Funct. Anal. 177 (2000), 282-309.

[5] G. Gripenberg, 'A necessary and sufficient condition for the existence of a father wavelet', Studia Math. 114 (1995), 207-226.

[6] Y-H. Ha, H.B. Kang, J.S. Lee and J.K. Seo, 'Unimodular wavelets for $L^{2}$ and the Hardy space $H^{2}$, Michigan Math. J. 41 (1994), 345-361.

[7] E. Hernández, and G. Weiss, A first course on wavelets (CRC Press, Boca Raton, 1996). 
[8] R.-Q. Jia, 'Shift-invariant spaces and linear operator equations', Israel J. Math. 103 (1998), 258-288.

[9] H.O. Kim and J.K. Lim, 'Frame multiresolution analysis', Commun. Korean Math. Soc. $15(2000), 285-308$.

[10] H.O. Kim and J.K. Lim, 'On frame wavelets associated with frame multi-resolution analysis', Appl. Comput. Harmon. Anal. 10 (2001), 61-70.

[11] H.O. Kim and J.K. Lim, 'Applications of shift-invariant space theory to some problems of multi-resolution analysis of $L^{2}\left(\mathbb{R}^{d}\right)$ ', (preprint 2000), in Proc. Conf. Wavelet Anal. Appl. (International Press, Boston, 2001) (to appear).

[12] H.O. Kim, R.Y. Kim and J.K. Lim, 'Characterizations of biorthogonal wavelets which are associated with biorthogonal multiresolution analyses', Appl. Comput. Harmon. Anal. 11 (2001), 263-272.

[13] M. Papadakis, 'On the dimension functions of orthonormal wavelets', Proc. Amer. Math. Soc. 128 (2000), 2043-2049.

[14] X. Wang, The study of wavelets from the properties of their Fourier transforms, $\mathrm{Ph} . \mathrm{D}$. Thesis (Washington University, St. Louis, 1995).

Division of Applied Mathematics

\section{KAIST}

373-1 Kusong-Dong Yusong-Gu

Taejon 305-701

Korea

e-mail: hkim@ftn.kaist.ac.kr

rykim@ftn.kaist.ac.kr
CHiPS

KAIST

373-1 Kusong-Dong Yusong-Gu

Taejon 305-701

Korea

e-mail: jaekun@ftn.kaist.ac.kr 